\title{
Stationary sets added when forcing squares
}

\author{
Maxwell Levine $^{1}(D)$
}

Received: 28 November 2016 / Accepted: 23 January 2018 / Published online: 2 February 2018

(C) The Author(s) 2018. This article is an open access publication

\begin{abstract}
Current research in set theory raises the possibility that $\square_{\kappa,<\lambda}$ can be made compatible with some stationary reflection, depending on the parameter $\lambda$. The purpose of this paper is to demonstrate the difficulty in such results. We prove that the poset $\mathbb{S}(\kappa,<\lambda)$, which adds a $\square_{\kappa,<\lambda}$-sequence by initial segments, will also add non-reflecting stationary sets concentrating in any given cofinality below $\kappa$. We also investigate the CMB poset, which adds $\square_{\kappa}^{*}$ in a slightly different way. We prove that the $\mathrm{CMB}$ poset also adds non-reflecting stationary sets, but not necessarily concentrating in any cofinality.
\end{abstract}

Keywords Set theory $\cdot$ Forcing $\cdot$ Large cardinals

Mathematics Subject Classification 03E55

\section{Introduction}

The $\square_{\kappa}$ principle, pronounced "square kappa," was introduced by Jensen in order to study the fine structure of Gödel's constructible universe $L$ [3]. $\square_{\kappa}$ and similar principles imply the failure of many of the compactness properties entailed by large cardinals. For our purposes we focus on stationary reflection.

In the first section we will define the hierarchy of principles $\square_{\kappa,<\lambda}$ for $1<\lambda \leq \kappa^{+}$ and summarize some of the existing work on the extent to which $\square_{\kappa,<\lambda}$ impedes stationary reflection for different values of $\lambda$. Then we will define two posets: one denoted $\mathbb{S}(\kappa,<\lambda)$ that forces $\square_{\kappa,<\lambda}$ for any fixed $\lambda$, and another called the CMB

Maxwell Levine

maxwell.levine@univie.ac.at

1 Kurt Gödel Research Center for Mathematical Logic, Universität Wien, Vienna, Austria 
poset, denoted $\mathbb{C}$, that forces $\square_{\kappa}^{*}$. In the second section we will show that $\mathbb{S}(\kappa,<\lambda)$ adds non-reflecting stationary sets, and in the third section we will show that $\mathbb{C}$ adds a non-reflecting stationary set as well. In the last section, we will show that in the case of singular cardinals of uncountable cofinality, $\mathbb{C}$ is compatible with some stationary reflection.

\section{Preliminaries}

Definition 1 For an infinite cardinal $\kappa$ and a cardinal $\lambda$ such that $1<\lambda \leq \kappa^{+}$, $\left\langle\mathcal{C}_{\alpha}: \alpha<\kappa^{+}\right\rangle$is a $\square_{\kappa,<\lambda}$-sequence if for all $\alpha$ :

- $1 \leq\left|\mathcal{C}_{\alpha}\right|<\lambda$;

- $\mathcal{C}_{\alpha}$ consists of clubs in $\alpha$;

- for all clubs $C \in \mathcal{C}_{\alpha}$, ot $C \leq \kappa$;

- and for all clubs $C \in \mathcal{C}_{\alpha}$ and for all $\beta \in \lim C, C \cap \beta \in \mathcal{C}_{\beta}$.

Here $\lim C$ denotes the accumulation points in a club $C$. Although we discuss only limit ordinals $\alpha$ here, indexing the sequence for all $\alpha<\kappa^{+}$is a standard abuse of notation.

$\square_{\kappa, \lambda}$ is defined similarly, but where the first bullet point is replaced by $1 \leq\left|\mathcal{C}_{\alpha}\right| \leq \lambda$. To say that $\square_{\kappa,<\lambda}$ holds is, naturally, to assert the existence of a $\square_{\kappa,<\lambda}$-sequence. Jensen's original $\square_{\kappa}$ is $\square_{\kappa, 1}$ while $\square_{\kappa, \kappa}$, which is usually denoted $\square_{\kappa}^{*}$, is called "weak square". The generalized definition for different values of $\lambda$ is due to Schimmerling [6].

The intuitive purpose for $\square_{\kappa,<\lambda}$ is to define a way of comparing a given model of set theory and $L$ - the smaller the value of $\lambda$, the more a given model resembles $L$. We are interested in the tension between $\square_{\kappa,<\lambda}$ for various $\lambda$ and stationary reflection, which is a key ingredient to Jensen's proof that $L$ contains Suslin trees and has numerous other applications.

Definition 2 If $\kappa$ is a regular cardinal and $S \subset \kappa$ is stationary in $\kappa$, then we say $S$ reflects at $\alpha \in \kappa$ if $\alpha$ has uncountable cofinality and $S \cap \alpha$ is stationary as a subset of $\alpha$.

Usually the assumption that $\alpha$ has uncountable cofinality is tacit. In general, $\square_{\kappa,<\lambda}$ clashes with stationary reflection. Some results along these lines follow:

Facts 1 [1]

(1) If $\square_{\kappa}$ holds then every stationary $S \subset \kappa^{+}$contains a non-reflecting stationary subset.

(2) If $\kappa$ is singular and $\square_{\kappa, \lambda}$ holds for $\lambda<\kappa$, then for every stationary $S \subset \kappa^{+}$, there is a sequence $\left\langle T_{i}: i<\mathrm{cf} \kappa\right\rangle$ of stationary subsets of $S$ so that for every $\alpha<\kappa^{+}$, there is some $i<\mathrm{cf} \kappa$ such that $T_{i}$ does not reflect at $\alpha$.

(3) Assuming the existence of countably many supercompact cardinals, it is consistent for $\kappa$ to be a singular cardinal of countable cofinality and for $\square_{\kappa, \omega}$ to hold while every stationary $S \subset \kappa^{+}$reflects to some $\alpha<\kappa^{+}$. 
(4) Again, assuming the existence of countably many supercompact cardinals: It is consistent for $\kappa$ to be a singular cardinal of countable cofinality and for $\square_{\kappa}^{*}$ to hold while for $\lambda<\kappa$ every sequence $\left\langle S_{i}: i<\lambda\right\rangle$ of stationary subsets of $\kappa^{+}$ containing ordinals of cofinality less than some $\tau<\kappa$ reflects simultaneously to some $\alpha<\kappa^{+}$, i.e. such that $S_{i} \cap \alpha$ is stationary for every $i<\lambda$.

These consistency results use variations on the following poset, which is used to force general square sequences.

Definition 3 For $2 \leq \lambda \leq \kappa^{+}$, let $\mathbb{S}(\kappa,<\lambda)$ be the poset of functions $p$ such that:

- $\operatorname{dom} p=\{\beta \leq \alpha: \lim \beta\}$ for some $\alpha \in \lim \kappa^{+}$;

- for all $\alpha \in \operatorname{dom} p, p(\alpha)$ is a set of clubs in $\alpha$ and $1 \leq|p(\alpha)|<\lambda$;

- for all $\alpha \in \operatorname{dom} p$ and every $C \in p(\alpha)$, ot $C \leq \kappa$;

- and for all $\alpha \in \operatorname{dom} p$, every $C \in p(\alpha)$, and every $\beta \in \lim C, C \cap \beta \in p(\beta)$.

The ordering is direct extension: $p \leq q$ if $p \uparrow \operatorname{dom} q=q \cdot \mathbb{S}(\kappa, \lambda)$ is defined similarly.

Fact $2[1] \mathbb{S}(\kappa,<\lambda)$ is $(\kappa+1)$-strategically closed and $\Vdash_{\mathbb{S}(\kappa,<\lambda)} \square_{\kappa,<\lambda}$.

A similar poset was recently studied by the author.

Definition 4 Let $\kappa$ be a singular strong limit of cofinality $\mu$ and let $\left\langle\kappa_{i}: i<\mu\right\rangle$ be a sequence of regular cardinals converging to $\kappa . \mathbb{C}_{i}$ is the poset of closed bounded subsets of $\kappa^{+}$of order-type less than $\kappa_{i}$, where $p \leq \mathbb{C}_{i} q$ if $p$ end-extends $q$, meaning that $\max p \geq \max q$ and $p \cap(\max q)=q$.

We let $\mathbb{C}=\prod_{i<\mu} \mathbb{C}_{i} / \sim$ where $f \sim g$ if $\exists j<\mu$ such that $i \geq j$ implies $f(i)=g(i)$. We use $[f]$ to refer to the equivalence class of $f .[f] \leq[g]$ will refer to ordering modulo the equivalence relation, and $f(i) \leq g(i)$ will refer to the ordering of $\mathbb{C}_{i}$. We call $\mathbb{C}$ the collapses-mod-bounded poset, or more briefly the CMB poset.

Facts 3 [5] Let $\mathbb{C}$ refer to the CMB poset.

(1) $\mathbb{C}$ is $(\kappa+1)$-strategically closed.

(2) $\mathbb{C}$ adds $\square_{\kappa}^{*}$.

(3) If $\tau$ is supercompact and $\mathbb{C}$ is defined in terms of a singular $\kappa>\tau$ with $\mathrm{cf} \kappa<\tau$, then $V^{\mathbb{C}}$ contains no very good scales on $\kappa$.

Although the isomorphism-type of $\mathbb{C}$ depends on the sequence $\left\langle\kappa_{i}: i<\mu\right\rangle$ used in its definition, the properties discussed will not depend on the particular sequence.

\section{Non-reflecting stationary sets added by $\mathbb{S}(\kappa,<\lambda)$}

For this section we fix an infinite cardinal $\kappa$ and a cardinal $\lambda$ such that $1<\lambda \leq \kappa^{+}$. We begin with a useful lemma, which shows that if $X$ is a set of constant cofinality in $\kappa^{+}$, then we can find an ordinal in $\kappa^{+}$in which to simultaneously embed all of the order types found in $X$. 
Lemma 1 Suppose $\kappa$ is a cardinal and $X \subset(\kappa+1) \cap \operatorname{cof}(\mu):=\{\alpha \leq \kappa: \operatorname{cf} \alpha=\mu\}$ for some regular $\mu \leq \kappa$. Then there is a $\rho<\kappa^{+}$and a collection $\left\langle C_{\alpha}: \alpha \in X\right\rangle$ of clubs in $\rho$ such that for every $\alpha \in X$, ot $C_{\alpha}=\alpha$. If $\mu=\operatorname{cf} \kappa$ or if $X$ is bounded in $\kappa$ then we can find $\rho \leq \kappa$ such that this works.

Proof If $\operatorname{cf} \kappa=\mu$ we let $\rho=\kappa$. Otherwise we let $\rho$ be the ordinal product $\sup X \cdot \mu$, noting that if $\sup X<\kappa$ then $\rho \leq \kappa$.

Fix $\alpha \in X$. If $\alpha=\kappa$, then $\mathrm{cf} \kappa=\mu$ and we simply let $C_{\alpha}=\kappa$. If not we define a continuous and cofinal function $f: \alpha \rightarrow \rho$ which allows us to define $C_{\alpha}$ as $\{f(\beta): \beta<\alpha\}$. We need a sequence $\left\langle\delta_{\xi}: \xi<\mu\right\rangle$ cofinal in $\rho$ such that $\delta_{\xi}+\alpha \leq \delta_{\xi+1}$ for all $\xi<\mu$. If $\mathrm{cf} \kappa=\mu$, we can obtain $\left\langle\delta_{\xi}: \xi<\mu\right\rangle$ because $\delta+\alpha<\kappa$ for any $\delta<\kappa$, and otherwise we obtain $\langle\delta \xi: \xi<\mu\rangle$ by letting $\delta_{\xi}=\sup X \cdot \xi$.

Let $\left\langle\gamma_{\xi}: \xi<\mu\right\rangle$ be continuous, increasing, and cofinal in $\alpha$ such that $\gamma_{0}=0$ and $\gamma_{\xi}$ is a successor ordinal if $\xi$ is a successor ordinal. Any ordinal $\gamma<\alpha$ is in the interval $\left[\gamma_{\xi}, \gamma_{\xi+1}\right)$ for some $\xi<\mu$, so it is enough to define $f$ on this interval. If $\gamma=\gamma_{\xi}+\beta<\gamma_{\xi+1}$ then let $f(\gamma)=\delta_{\xi}+\beta$. Then $f$ is clearly increasing and it is cofinal in $\rho$ because the sequence of $\delta \xi$ 's is cofinal in $\rho$.

It remains to show that $f$ is continuous. Suppose $\gamma<\alpha$ is a limit. If $\gamma=\gamma_{\xi}$, then by construction $\xi$ is a limit ordinal for some $\xi<\mu$ and so

$$
f(\gamma)=\delta_{\xi}=\sup _{\eta<\xi} \delta_{\eta}=\sup _{\eta<\xi} f\left(\gamma_{\xi}\right)=\sup \{f(\beta): \beta<\gamma\} .
$$

Otherwise $\gamma=\gamma_{\xi}+\beta$ for some limit $\beta>0$ and so

$$
f(\gamma)=\delta_{\xi}+\beta=\sup _{\beta^{\prime}<\beta}\left(\delta_{\xi}+\beta^{\prime}\right)=\sup _{\beta^{\prime}<\beta} f\left(\gamma_{\xi}+\beta^{\prime}\right)=\sup \{f(\beta): \beta<\gamma\} .
$$

Observe that if $X \subset(\kappa+1) \cap \operatorname{cof}(\mu)$ is unbounded in $\kappa$ and $\mu \neq \operatorname{cof} \kappa$, then it is impossible to obtain the conclusion of this lemma for any $\rho \leq \kappa$. Since sup $X=\kappa$ in this case, it follows that $\rho \geq \kappa$, and then since $\operatorname{cf} \rho \neq \operatorname{cf} \kappa$ we have $\rho>\kappa$.

Lemma 2 If $\mathbb{S}=\mathbb{S}(\kappa,<\lambda), q \in \mathbb{S}$, and $q \Vdash$ " $\dot{C}$ is a club in $\kappa^{+}$, then $\{p \in \mathbb{S}: p \Vdash$ " $\max \operatorname{dom} p \in \dot{C} "\}$ is dense below $q$.

Proof Build an increasing sequence of ordinals $\left\langle\alpha_{n}: n<\omega\right\rangle$ and a decreasing sequence of conditions $\left\langle p_{n}: n<\omega\right\rangle$ by induction, and for clarity let $\beta_{n}=$ $\max$ dom $p_{n}$. Pick $p_{0} \leq q$ and $\alpha_{0}<\kappa^{+}$such that $p_{0} \Vdash$ " $\alpha_{0} \in \dot{C}$ ". Given $\alpha_{n}, p_{n}$, choose $\alpha_{n+1}>\beta_{n}$ and $r \leq p_{n}$ such that $r \Vdash$ " $\alpha_{n+1} \in \dot{C}$ ". Then choose $p_{n+1} \leq r$ so that $\beta_{n+1}=\max \operatorname{dom} p_{n+1}>\alpha_{n+1}$, so we still have $p_{n+1} \Vdash$ " $\alpha_{n+1} \in \dot{C}$ ".

Let $\gamma=\sup _{n<\omega} \alpha_{n}$, and note that $\gamma=\sup _{n<\omega} \beta_{n}$ as well because $\alpha_{n} \leq \beta_{n}<\alpha_{n+1}$ for all $n<\omega$. Define $p$ such that $p\left\lceil\beta_{n}=p_{n}\right.$ for all $n<\omega$ and $p(\gamma)=\left\{\left\{\beta_{n}: n<\omega\right\}\right\}$ (any $\omega$-sequence will do), so $\max \operatorname{dom} p=\gamma$. Then $p$ is a condition stronger than the $p_{n}$ 's, so $p \Vdash$ " $\alpha_{n} \in \dot{C}$ " for all $n<\omega$ and then since $p \Vdash$ " $\dot{C}$ is a club" it follows that $p \Vdash$ " $\gamma \in \dot{C}$ ". 
Definition 5 Consider $\mathbb{S}(\kappa,<\lambda)$ and let $\dot{\mathcal{C}}_{\alpha}$ be a canonical name for the $\alpha$ th level of the generic square sequence. In other words, if $\alpha \in \operatorname{dom} p$ then $p \Vdash$ " $\dot{\mathcal{C}}_{\alpha}=p(\alpha)$ ". If $X \subset \kappa+1$, let $\dot{S}_{X}$ be the canonical $\mathbb{S}(\kappa,<\lambda)$-name such that $\vdash_{\mathbb{S}(\kappa,<\lambda)}$ " $\dot{S}_{X}=\{\alpha<$ $\kappa^{+}:\left\{\right.$ot $\left.\left.C: C \in \dot{\mathcal{C}}_{\alpha}\right\}=X\right\}$ ".

Theorem 1 If $\mu \leq \kappa$ is regular, $X$ is a non-empty subset of $(\kappa+1) \cap \operatorname{cof}(\mu)$ of size less than $\lambda$, and either $X$ is bounded in $\kappa$ or $\operatorname{cf} \kappa=\mu$, then $\Vdash_{\mathbb{S}(\kappa,<\lambda)}$ " $\dot{S}_{X}$ is stationary in $\kappa^{+}$".

Proof Apply Lemma 1 to $X$ in order to obtain $\delta \leq \kappa$ and clubs $C_{\alpha} \subset \delta$ such that ot $C_{\alpha}=\alpha$ for all $\alpha \in X$. Let $q \in \mathbb{S}(\kappa,<\lambda)$ be a condition forcing that $\dot{C}$ is a club in $\kappa^{+}$. We will construct a descending sequence $\left\langle p_{\xi}: \xi \leq \delta\right\rangle$ below $q$ where $\gamma_{\xi}=\max \operatorname{dom} p_{\xi}$ as follows:

- Find $\alpha_{0}$ and $r \leq q$ such that $r \Vdash$ " $\alpha_{0} \in \dot{C}$ ". Then find $p_{0} \leq r$ such that $\max \operatorname{dom} p_{0}>\alpha_{0}$.

- Similarly for successors, choose $p_{\xi+1} \leq p_{\xi}$ such that $\gamma_{\xi+1}>\gamma_{\xi}$ and $p_{\xi+1} \Vdash$ " $\gamma_{\xi+1} \in \dot{C}$ " using Lemma 2.

- Suppose $\xi<\delta$ is a limit. Let $p_{\xi}\left\lceil\gamma_{\eta}=p_{\eta}\right.$ for $\eta<\xi$. If $\alpha \in Y:=\{\alpha \in X$ : $C_{\alpha}$ is unbounded in $\left.\xi\right\}$, then let $D_{\alpha}=\left\{\gamma_{\eta}: \eta \in C_{\alpha} \cap \xi\right\}$. Also define $D=\left\{\gamma_{\eta}\right.$ : $\eta<\xi\}$ (in case $Y$ is empty). Then let $\gamma_{\xi}=\sup _{\eta<\xi} \gamma_{\eta}$ and let $p_{\xi}\left(\gamma_{\xi}\right)=\left\{D_{\alpha}: \alpha \in\right.$ $Y\} \cup\{D\}$.

We must show that $p_{\xi}$ is a condition in $\mathbb{S}(\kappa,<\lambda)$. The only nontrivial consideration is to show that if $E \in p_{\xi}\left(\gamma_{\xi}\right)$ and $\beta \in \lim E$, then $E \cap \beta \in p_{\xi}(\beta)$. If $\beta \in$ $\lim E$ then $\beta=\gamma_{\zeta}$ such that $\zeta$ is a limit. If $E=\left\{\gamma_{\eta}: \eta<\xi\right\}$ then this means $E \cap \beta=\left\{\gamma_{\eta}: \eta<\zeta\right\}$, so this club would have been included at step $\zeta$ in the definition of $p_{\zeta}$. If $E=\left\{\gamma_{\eta}: \eta \in C_{\alpha} \cap \xi\right\}$ for some $\alpha \in Y$, then this means that $\left\{\gamma_{\eta}: \eta \in C_{\alpha} \cap \zeta\right\}$ is unbounded in $\gamma_{\zeta}$ and therefore that $C_{\alpha}$ is unbounded in $\zeta$, so the club $E \cap \beta=\left\{\gamma_{\eta}: \eta \in C_{\alpha} \cap \zeta\right\}$ would have been included at step $\zeta$.

- Suppose $\xi=\delta$. This is the same as the other limit cases except that $p_{\delta}\left(\gamma_{\delta}\right)=\left\{D_{\alpha}\right.$ : $\alpha \in X\}$ and we know that $\left\{\alpha \in X: C_{\alpha}\right.$ is unbounded in $\left.\delta\right\}=X$. Showing that $p_{\delta}$ is a condition is strictly simpler than in the previous case.

It follows that $p_{\delta} \Vdash$ " $\gamma_{\delta} \in \dot{C} \cap \dot{S}_{X}$ ".

When using a generalized $\square_{\kappa,<\lambda}$-sequence $\left\langle\mathcal{C}_{\alpha}: \alpha<\kappa^{+}\right\rangle$to prove a non-reflection result, it is typical to make use of the function $F: \alpha \mapsto\left\{\right.$ ot $\left.C: C \in \mathcal{C}_{\alpha}\right\}$ and to find a stationary set on which $F$ is constant. The significance of the above theorem is that

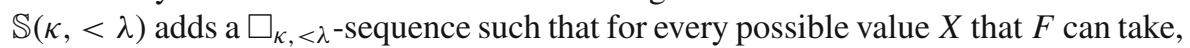
$F^{-1}(X)$ is stationary.

Lemma 3 If $\delta \leq \kappa$, then $\Vdash_{\mathbb{S}(\kappa,<\lambda)}$ “ $\dot{S}_{\{\delta\}}$ is non-reflecting”.

Proof Work in $V^{\mathbb{S}(\kappa,<\lambda)}$ and suppose for contradiction that $S_{\{\delta\}}$ reflects at $\alpha \in \kappa^{+} \cap$ $\operatorname{cof}(>\omega)$. If $C \in \mathcal{C}_{\alpha}$ (chosen from the generically added sequence), choose $\beta<\gamma<\alpha$ such that $\beta, \gamma \in \lim C \cap S_{\{\delta\}}$. Then $\delta=\operatorname{ot}(C \cap \beta)<\operatorname{ot}(C \cap \gamma)=\delta$, a contradiction. $\square$

Corollary $1 \mathbb{S}(\kappa,<\lambda)$ adds non-reflecting stationary subsets of $\kappa^{+} \cap \operatorname{cof}(\mu)$ for every regular $\mu \leq \kappa$. 


\section{The non-reflecting stationary set added by the CMB poset}

For the remainder of this paper, fix a singular strong limit cardinal $\kappa$ of cofinality $\mu$ and a sequence $\left\langle\kappa_{i}: i<\mu\right\rangle$ of regular cardinals converging to $\kappa$, and define the CMB poset $\mathbb{C}$ in terms of this sequence as in Definition 4 . Using similar methods to the previous section, we prove:

Theorem $2 \mathbb{C}$ adds a non-reflecting stationary set in $\kappa^{+} \cap \operatorname{cof}(\mu)$.

Proof Let $G$ be $\mathbb{C}$-generic. Elements of $G$ are denoted by equivalence classes $[f]$ of representatives $f \in \prod_{i<\mu} \mathbb{C}_{i}$. Working in $V[G]$, we define

$$
X:=\left\{\alpha<\kappa^{+}: \exists[f] \in G, j<\mu \text { s.t. } i \geq j \Longrightarrow \alpha \in \lim f(i)\right\} .
$$

Observe that the definition of $X$ does not depend on the representative. We will make use of the following fact, that was earlier established by the author [5]:

Fact 4 If $\alpha<\kappa^{+}$is a limit ordinal such that $\sup (X \cap \alpha)=\alpha$ and $\operatorname{cf} \alpha \neq \mu$, then $\alpha \in X$.

The non-reflecting stationary set will be

$$
S:=\left\{\alpha<\kappa^{+}: \sup (X \cap \alpha)=\alpha \text { but } \alpha \notin X\right\} .
$$

Note that Fact 4 implies that $S \subset \kappa^{+} \cap \operatorname{cof}(\mu)$.

Claim $1 S$ is stationary in $\kappa^{+}$.

Proof Work in $V$ and suppose [f] $\Vdash \dot{C}$ is a club in $\kappa^{+}$". Let $\dot{X}$ and $\dot{S}$ be canonical names for $X$ and $S$. Our goal is to find an $[h] \leq[f]$ so that $[h] \Vdash " \dot{C} \cap \dot{S} \neq \emptyset$ ".

We will construct a descending sequence of conditions $\left\langle\left[f_{\xi}\right]: \xi<\mu\right\rangle$, choosing representatives $f_{\xi}$ as we go, and an ascending sequence of ordinals $\left\langle\alpha_{\xi}: \xi<\mu\right\rangle$. Denote $\gamma_{\xi}=\sup _{i<\mu} \max f_{\xi}(i)<\kappa^{+}$. We will require that: $f_{\xi}(i)=\emptyset$ for all $i<$ $\xi<\mu$ (i.e. $f_{\xi}(i)$ is the weakest condition in $\left.\mathbb{C}_{i}\right) ; \gamma_{\xi} \in f_{\xi}(i)$ for all $\xi \leq i<\mu$; $f_{\xi}(i) \leq f_{\eta}(i)$ for all $\eta \leq \xi \leq i<\mu$; and $\alpha_{\xi} \leq \gamma_{\xi}<\alpha_{\xi+1}$ for all $\xi<\mu$.

Let $\left[f_{0}\right] \leq[f]$ be a condition such that $\left[f_{0}\right] \Vdash$ " $\alpha_{0} \in \dot{C}$ " for some $\alpha_{0}<\kappa^{+}$. For successor cases, if we are given $\left[f_{\xi}\right], \alpha_{\xi}$, choose $[g] \leq\left[f_{\xi}\right]$ such that there is some $\alpha_{\xi+1}>\gamma_{\xi}$ such that $[g] \Vdash$ " $\alpha_{\xi+1} \in \dot{C}$ ". Then choose $f_{\xi+1}$ such that: $\gamma_{\xi+1}=$ $\sup _{i<\mu} \max f_{\xi+1}(i)>\alpha_{\xi+1} ; \gamma_{\xi+1} \in f_{\xi+1}(i)$ for $i \geq \xi+1 ;\left[f_{\xi+1}\right] \leq[g] ; f_{\xi+1}(i) \leq$ $f_{\xi}(i)$ for all $i \geq \xi+1$; and $f_{\xi+1}(i)=\emptyset$ for $i<\xi+1$. If $\xi$ is a limit then let $\alpha_{\xi}=$ $\sup _{\eta<\xi} \alpha_{\eta}$, let $f_{\xi}(i)=\emptyset$ for $i<\xi$, and let $f_{\xi}(i)=\bigcup_{\eta<\xi} f_{\eta}(i) \cup\left\{\sup _{\eta<\xi} \max f_{\eta}(i)\right\}$ for $i \geq \xi$. In this case $\alpha_{\xi}=\gamma_{\xi}$ by interleaving and $\left[f_{\xi}\right] \Vdash$ " $\alpha_{\xi} \in \dot{C}$ " because $\dot{C}$ is forced to be a club.

Again by interleaving, $\sup _{\xi<\mu} \alpha_{\xi}=\sup _{\xi<\mu} \gamma_{\xi}$, so call this ordinal $\beta$. Let $f^{*}$ be a function so that $f^{*}(i)=f_{i}(i)$. Then $\forall \xi<\mu, \xi \leq i, f^{*}(i) \leq f_{\xi}(i)$, so $\left[f^{*}\right] \leq\left[f_{\xi}\right]$ for all $\xi<\mu$. Hence $\left[f^{*}\right] \Vdash$ " $\beta \in \dot{C} "$. Now choose $\delta>\beta$ and define $h$ such that $h(i)=f^{*}(i) \cup\{\delta\}$. 
We have $[h] \leq\left[f^{*}\right]$, so $[h] \Vdash$ " $\beta \in \dot{C}$ ". Furthermore, $\forall \xi<\mu, \gamma_{\xi} \in f_{\xi}(i)$ for $i \geq \xi$, so $[h] \Vdash$ “ $\gamma_{\xi} \in X$ ". Hence, $[h] \Vdash$ " $X$ is unbounded in $X \cap \beta$ ". On the other hand, $h(i) \cap\left(\gamma_{i}, \delta\right)=\emptyset$ for all $i$. That means that if $[g] \leq[h]$ and $j$ is such that $i \geq j$ implies that $g(i) \leq h(i)$, then it follows that $\beta \notin g(i)$ for any $i \geq j$. Hence $[h] \Vdash " \beta \notin X "$. Therefore we have shown that $[h] \Vdash " \dot{C} \cap \dot{S} \neq \emptyset$ ".

Claim $2 S$ does not reflect at any $\alpha<\kappa^{+}$of uncountable cofinality. In other words, $S$ is a non-reflecting stationary set.

Proof Work in $V[G]$ and fix some $\alpha<\kappa^{+}$of uncountable cofinality. We will show that $S$ cannot reflect at $\alpha$. Suppose for contradiction that it does.

Since $X$ is unbounded in every point in $S, X$ is unbounded in $\alpha$. The fact that $S \subset \kappa^{+} \cap \operatorname{cof}(\mu)$ implies that $\operatorname{cf} \alpha>\mu$. It follows from Fact 4 that $\alpha \in X$. Now let $j<\mu$ be such that $\alpha \in \lim f(i)$ for all $i \geq j$ and let $C=\alpha \cap \bigcap_{i \geq j} f(i)$. Since cf $\alpha>\mu, C$ is a club in $\alpha$. Moreover, if $\beta \in \lim C$, then clearly $\beta \in X$, and since $X \cap S=\emptyset$ by definition, we have $\lim C \cap S=\emptyset$.

This completes the proof.

\section{Reflection in extensions by the CMB poset}

In this section we demonstrate that the CMB poset $\mathbb{C}$ does not necessarily add nonreflecting stationary sets in all cofinalities. We must draw on several facts from the literature. The first is widely known:

Fact 5 If $\lambda$ is supercompact, $v>\lambda$ is regular, and $S \subset v \cap \operatorname{cof}(<\lambda)$ is stationary, then $S$ reflects.

The second fact is a result of Laver [4].

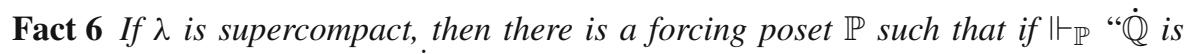
$\lambda$-directed closed", then $V^{\mathbb{P} * \dot{\mathbb{Q}}} \models$ " $\lambda$ is supercompact".

A supercompact cardinal having this property is called "indestructibly supercompact".

Finally, we use a fact drawn from the theory of the approachability ideal, namely that $\square_{\kappa}^{*}$ implies the approachability property at $\kappa$, which in turn implies that stationary subsets of $S \subset \kappa^{+} \cap \operatorname{cof}(\tau)$ are preserved by $\tau^{+}$-closed forcing posets [2].

Fact 7 If $\square_{\kappa}^{*}$ holds for a singular cardinal $\kappa$ and $S \subset \kappa^{+} \cap \operatorname{cof}(\tau)$ is stationary for some regular $\tau<\kappa$, then $S$ is stationary in any $\tau^{+}$-closed forcing extension.

Theorem 3 Suppose $\lambda$ is indestructibly supercompact and $\omega<\mu<\lambda<\kappa$. Then in $V^{\mathbb{C}}$, if $\tau<\mu$ then every stationary subset of $\kappa^{+} \cap \operatorname{cof}(\tau)$ reflects.

Proof Fix a $\mathbb{C}$-generic filter $G$ and a stationary set $S \subset \kappa^{+} \cap \operatorname{cof}(\tau)$ such that $S \in$ $V[G]$. Let $j$ be such that $\tau, \lambda<\kappa_{j}$ and let $\mathbb{D}:=\prod_{j \leq i<\mu} \mathbb{C}_{i}$, where $\mathbb{C}_{i}$ is again the set of closed bounded sets in $\kappa^{+}$of order-type less than $\kappa_{i}$, and $\mathbb{C}_{i}$ is ordered by 
end-extension. Observe that $\mathbb{D}$ is $\kappa_{j}$-directed closed since the same is true of each $\mathbb{C}_{i}$ for $i \geq j$. If $e(i)=\emptyset$ for $i<j$ let $\pi(f)=\left[e^{\frown f}\right] \in \mathbb{C}$. Observe that $\pi: \mathbb{D} \rightarrow \mathbb{C}$ is a projection of forcing posets in the sense that if $\mathbb{D} / G:=\{f \in \mathbb{D}: \pi(f) \in G\}$ and $H$ is $\mathbb{D} / G$-generic, then there is a $\mathbb{D}$-generic $K$ such that $V[G * H]=V[K]$.

Claim $3 \mathbb{D} / G$ is $\mu$-closed.

Proof Suppose $\left\langle f_{\xi}: \xi<\eta\right\rangle \subset \mathbb{D} / G$ is a descending sequence for $\eta<\mu$, and let $g_{\xi}$ witness that $f_{\xi} \in \mathbb{D} / G$, meaning that $\pi\left(f_{\xi}\right) \in G$. Then $D_{\xi}:=\{[g] \in \mathbb{C}:[g] \leq$ $\left[f_{\xi}\right]$ or $\left.[g] \perp\left[f_{\xi}\right]\right\}$ is open dense. Since $\mathbb{C}$ is $\kappa^{+}$-distributive, $\left\langle D_{\xi}: \xi<\eta\right\rangle \in V$ and $D:=\bigcap_{\xi<\eta} D_{\xi}$ is open dense in $V$, so there is some $g \in \prod_{i<\mu} \mathbb{C}_{i}$ so that $[g] \in D \cap G$, which means $[g] \leq \pi\left(f_{\xi}\right)$ for all $\xi<\eta$. This in turn means that for every $\xi<\eta$ there is some $j \leq i_{\xi}<\mu$ such that for $i \geq i_{\xi}, g(i) \leq f_{\xi}(i)$. Let $i^{*}=\sup _{\xi<\eta} i_{\xi}$, so $i^{*}<\mu$ by regularity. Now define $f(i)=\bigcup_{\xi<\eta} f_{\xi}(i) \cup\left\{\sup _{\xi<\eta} \max f_{\xi}(i)\right\}$ for all $j \leq i<\mu$. It is immediate that $f \leq f_{\xi}$ for all $\xi<\eta$ in the ordering of $\mathbb{D}$. Furthermore, for all $i \geq i^{*}, g(i) \leq f(i)$. Hence we have that $[g] \leq \pi(f)$, so $\pi(f) \in G$ and thus $f \in \mathbb{D} / G$.

If $H$ is $\mathbb{D} / G$-generic, it follows from Fact 7 that $V[G * H] \models$ " $S$ is stationary in $v$ ". Then, since $\mathbb{D}$ is $\kappa_{j}$-directed closed and $\lambda<\kappa_{j}$, Fact 6 implies that $V[G * H] \models$ " $\lambda$ is supercompact. And so Fact 5 tells us that $V[G * H] \models$ " $S$ reflects". Reflection is downward absolute, so $V[G] \models$ " $S$ reflects".

In this paper we provided a poset $\mathbb{S}(\kappa,<\lambda)$ that adds non-reflecting stationary subsets of $\kappa^{+}$in any cofinality, and then a poset $\mathbb{C}$ that adds non-reflecting stationary subsets of $\kappa^{+}$in the critical cofinality cf $\kappa$ but not necessarily in cofinalities less than cf $\kappa$. But what about cofinalities greater than $\mathrm{cf} \kappa$ ? We close with a question.

Question 1 If the CMB poset is defined in terms of a singular strong limit $\kappa$ with cf $\kappa=\mu$, then does it add non-reflecting stationary subsets of $\kappa^{+} \cap \operatorname{cof}(\tau)$ for $\tau>\mu$ ?

Acknowledgements Open access funding provided by University of Vienna.

Open Access This article is distributed under the terms of the Creative Commons Attribution 4.0 International License (http://creativecommons.org/licenses/by/4.0/), which permits unrestricted use, distribution, and reproduction in any medium, provided you give appropriate credit to the original author(s) and the source, provide a link to the Creative Commons license, and indicate if changes were made.

\section{References}

1. Cummings, J., Foreman, M., Magidor, M.: Squares, scales, and stationary reflection. J. Math. Log. 1, 35-98 (2001)

2. Eisworth, T.: Successors of singular cardinals. In: Foreman, M., Kanamori, A. (eds.) Handbook of Set Theory, pp. 1229-1350. Springer, Dordrecht (2010)

3. Jensen, R.: The fine structure of the constructible hierarchy. Ann. Math. Log. 4, 229-308 (1972)

4. Laver, R.: Making the supercompactness of $\kappa$ indestructible under $\kappa$-directed closed forcing. Isr. J. Math. 29, 385-388 (1978)

5. Levine, M.: Weak squares and very good scales (2016, submitted)

6. Schimmerling, E.: Combinatorial principles in the core model for one Woodin cardinal. Ann. Pure Appl. Log. 74, 153-201 (1995) 